\title{
Insight into single-fiber push-out test of tungsten fiber-reinforced tungsten
}

\author{
Stephan Schönen ${ }^{\mathrm{a}}$, Bruno Jasper ${ }^{\mathrm{b}}$, Jan Willem Coenen ${ }^{\mathrm{b}}$, Juan $\mathrm{Du}^{\mathrm{b}}$, Till Höschen ${ }^{\mathrm{c}}$, \\ Johann Riesch ${ }^{\mathrm{c}}$, Ghaleb Natour ${ }^{\mathrm{a}}$, Rudolf $\mathrm{Neu}^{\mathrm{c}, \mathrm{d}}$, Christian Linsmeier ${ }^{\mathrm{b}}$ \\ ${ }^{a}$ Forschungszentrum Jülich GmbH, Zentralinstitut für Engineering, Elektronik und Analytik \\ - Engineering und Technologie, 52425 Jülich, Germany; \\ ${ }^{\mathrm{b}}$ Forschungszentrum Jülich GmbH, Institut für Energie- und Klimaforschung - Plasmaphysik, \\ 52425 Jülich, Germany; \\ ${ }^{\mathrm{c}}$ Max-Planck-Institut für Plasmaphysik, Boltzmannstr. 2, 85748 Garching, Germany; \\ dTechnische Universität München, Boltzmannstrasse 15, 85748 Garching, Germany
}

\section{ARTICLE HISTORY}

Compiled May 9, 2018

\begin{abstract}
To overcome the intrinsic brittleness of tungsten (W), a tungsten fiber-reinforced tungsten-composite material $\left(\mathrm{W}_{\mathrm{f}} / \mathrm{W}\right)$ is a possible solution. The introduction of energy dissipation mechanisms like fiber bridging or fiber pull-out by means of an engineered interface between fiber and matrix mitigate the brittleness of tungsten and lead to a pseudo-ductile material behaviour. The push-out test of single-fiber samples is an experimental method to investigate the properties of the interface between fiber and matrix of composite materials. It is widely used for the investigation of ceramic composites. This method was also used to investigate the debonding and frictional properties of the $\mathrm{Er}_{2} \mathrm{O}_{3}$ interface region between fiber and matrix of $\mathrm{W}_{\mathrm{f}} / \mathrm{W}$ single-fiber samples made by CVD- and HIP-processes. In this article finite element calculations are used to get a better understanding of the processes acting in the interface during a push-out test of $\mathrm{W}_{\mathrm{f}} / \mathrm{W}$. A detailed overview of the debonding progress and of the corresponding stress states of the interface during the different stages of the test is presented. In addition the sensitivity of the push-out behaviour regarding the different interface properties and the plastic flow curve of the tungsten fiber are investigated.
\end{abstract}

\section{KEYWORDS}

Metal-matrix composites (MMCs); fiber/matrix bond; cohesive interface modelling; finite element analysis (FEA); push-out test; tungsten fiber-reinforced tungsten composites

\section{Introduction}

It is expected that the required properties of materials for fusion power plants are beyond the technical limits of materials that are available today [1]. Tungsten is currently the main candidate material for plasma-facing components of future fusionreactors. It is very resistant against erosion, has the highest melting point of all metals and shows low hydrogen retention [2-4]. Besides this bulk tungsten material suffers from inherent brittleness below the ductile-to-brittle transition temperature (DBTT) [5]. Neutron irradiation during operation of the reactor can lead to further embritt- 
lement [6]. This lack of intrinsic toughness can be addressed by introducing extrinsic mechanisms of energy dissipation (extrinsic toughening) [7].

\section{Tungsten fiber-reinforced tungsten $\left(\mathrm{W}_{\mathrm{f}} / \mathrm{W}\right)$}

One approach for introducing extrinsic toughening is to reinforce the bulk material with fibers in combination with an engineered interface [8]. This technique is well known from fiber-reinforced ceramic matrix composites (CMC) $[9,10]$. If cracks in the matrix occur, fibers in the crack will activate mechanisms of energy dissipation. These mechanisms reduce the stress intensity at the crack tip of the matrix. For the case of a brittle fiber in a brittle matrix the deformation energy is dissipated by creating new free surfaces due to the fracture of the interface, to the bridging and thus elastic loading of the fiber and friction during pull-out of broken fibers. For the case of reinforcement with fibers with inherent ductility, energy can additionally be dissipated by plastic deformation of the fiber. This is the case for tungsten fibers in an 'asfabricated condition' [11]. Thermal treatment during the production of the composite or through operation conditions of the fusion reactor and additional embrittlement due to neutron irradiation can reduce the ductility of the tungsten fibers [12].

The interface properties have to be in an appropriate range to achieve the desired toughening. If the interface is too strong no interface debonding will occur and fiber rupture without the activation of any mechanisms will be the consequence. A very weak interface leads to low bridging-forces which reduces the mitigation of the stresses at the crack tip $[7,10]$. Therefore the identification of the optimal interface properties plays an important role for a successful design of tungsten fiber-tungsten reinforced composite material.

\section{Interface properties of $W_{f} / W$}

The most important properties to characterise the interface between fiber and matrix are:

- shear strength: $\tau_{\max }$

- critical fracture energy (mode II): $G_{2 C}$

- coefficient of friction after debonding: $\mu$

- initial interface pressure: $p_{i n i}$

While shear strength, critical fracture energy and coefficient of friction are inherent properties of the interface, the initial interface pressure is a result of the interaction between matrix, interface, fiber and the production process. For multi-materialcomposites the initial interface pressure can be explained by residual stresses that are induced by different thermal expansions of fiber and matrix during the production process. Fiber-reinforced plastics (FRP) with a matrix made of thermosetting polymers like epoxy resin can show initial interface pressure due to shrinking of the matrix as a result of the chemical cross-link polymerization reaction during the curing of the resin [13]. In [11] it was expected that growth stresses caused by the CVD-process (chemicalvapour-deposition) will be low. Another explanation is based on the assumption that the interface pressure is not initially present but develops as soon as relative sliding between fiber and matrix occurs. It is assumed that it is caused by geometrical irregularities of the fibers [14]. However, the experimentally derived force-displacement 
curves of push-out tests of Du [14] and Jasper [15] lead to the conclusion that an interface pressure is somehow present during the frictional phase of the experiments after the completion of interface debonding.

The single-fiber push-out test is one of the most popular methods to investigate the interface properties of fiber-reinforced composite materials (Figure 1) [16,17]. In the push-out test a thin slice of a single-fiber composite is placed onto a holder which contains a circular hole. The diameter of the hole is slightly larger than the one of the fiber to allow the push-through of the fiber. The fiber is then pushed from the top with a micro-indenter until debonding occurs. The push-out test needs little effort concerning practical implementation compared to other methods. The pull-out test, for example, which is closer to the situation in a real component where the fiber is pulled out in the crack wake, is much more challenging regarding specimen preparation.



Figure 1. Schematic of the set-up of fiber push-out test.

Analytical approaches are available to extract interface properties from the evaluated force-displacement relationship [16-19]. Therefore the common way to interpret the experimental results of the push-out tests is to apply these analytical models and to determine the associated parameters by a fitting procedure. These models obtain the required information from the force levels at the different stages of the tests. The stages of the test are identified by the qualitative shape of the experimental forcedisplacement curves.

However, these models have some restrictions that do not always reflect the experimental conditions. They include assumptions like purely elastic material behaviour without any plastic deformation of the fiber and the matrix. Furthermore they are not able to capture the influence of all environmental boundary conditions, e.g. the bending of the specimen due to the difference of the diameters of the fiber and the specimen holder is not taken into account. The shear lag model, which is commonly used to describe the push-out force during the frictional phase after the completion of debonding, is only valid for small relative displacements between fiber and matrix [17]. The well established finite element method (FEM) was applied to create a deeper understanding of the mechanisms that are acting during the different phases of a push-out test, accompanying the experimental work of Jasper [15].

Du investigated various single and multi-layer interfaces based on erbium oxide, zirconia oxide, carbon and copper [14,20,21]. The calculated shear strength of the oxides were comparable whereas the interfacial fracture energy of the coating with erbium oxide with a thickness of $600 \mathrm{~nm}$ deposited by magnetron sputtering was approximately three times higher than the one of zirconia oxide. Thus it is one of the most promising candidates as interface for $\mathrm{W}_{\mathrm{f}} / \mathrm{W}$. Furthermore Mao could show that reinforcing $\mathrm{W}_{\mathrm{f}} / \mathrm{W}$ samples produced by field assisted sintering technology (FAST) with discontinuous fibers coated with yttrium oxide $\left(\mathrm{Y}_{2} \mathrm{O}_{3}\right)$ results in significant toughening $[22]$. 


\section{Stages of a single-fiber push-out test}

Figure 2 shows a typical force-displacement curve of a push-out test as discussed by Chandra et al. [23]. The push-out test can be divided into three different stages:

- Stage I: elastic deformation

- Stage II: progress of debonding

- Stage III: frictional sliding

\subsection{Stage I: elastic deformation}

At the beginning of the test the specimen shows linear-elastic behaviour. The slope is the elastic bending stiffness of the specimen. The stress state of the interface is characterized by the distribution of normal pressure and shear stress. During this stage the interface pressure can be separated into components with different causes (Figure 3). The final stress profile results from the sum of the different components and depends on the elastic and geometrical properties of the specimen and the load applied by the indenter.

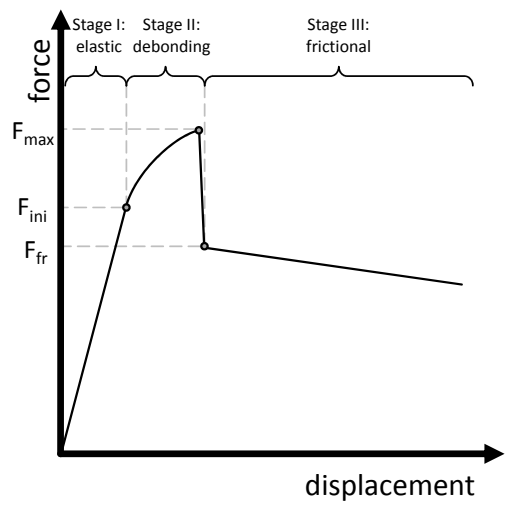

Figure 2. Typical force-displacement curve of a single-fiber push-out test according to Chandra et al. [23].
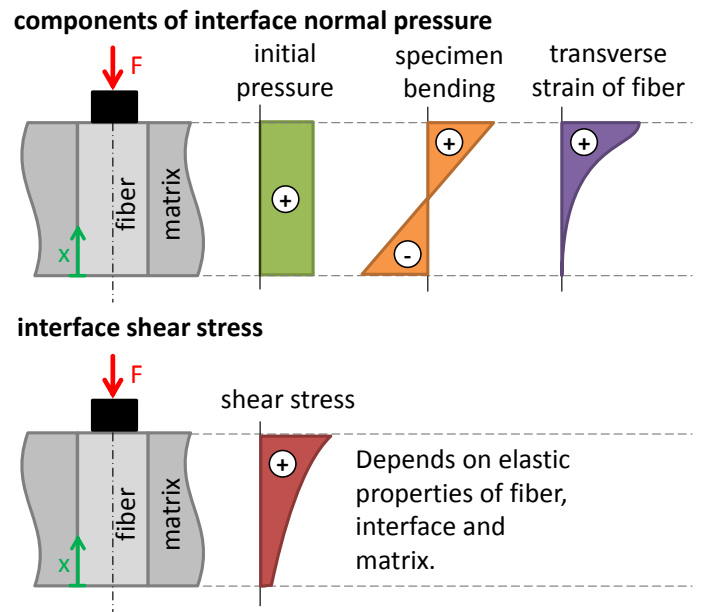

Figure 3. Schematic of the components of normal interface pressure and interface shear stress during the elastic phase (no debonding of the interface) of the push-out test. 
During the push-out test the specimen is placed on a holder containing a hole with typical diameters of $200 \mu \mathrm{m}-400 \mu \mathrm{m}$ for a fiber diameter of $150 \mu \mathrm{m}$. The difference of the diameters of indenter and the hole in the specimen holder leads to a bending of the specimen and the corresponding normal pressure with compression at the top and tension at the bottom of the specimen. This component increases for thinner specimens and larger hole diameters. The transverse strain of the fiber due to the poisson effect leads to an additional component of the interface pressure. Its maximum value is near the top of the specimen and it decreases with increasing distance to the top. In contrast, the shear stress distribution along the fiber length for the fully bonded case caused by the indenter force depends on the elastic properties of the fiber, interface and matrix and cannot as easily be split into different components as the normal pressure. For the case of a mismatch of the coefficients of thermal expansion between fiber and matrix the existence of an initial shear stress is also possible.

\subsection{Stage II: progress of debonding}

When the shear stress in the interface reaches its shear strength, debonding is initiated. Now the interface can be divided into a bonded and a debonded zone. Depending on the presence of normal pressure and the coefficient of friction the interface of the debonded zone is still able to transfer forces by frictional shear stresses. As shown in Figure 2 the stiffness of the specimen decreases during this stage.

\subsection{Stage III: frictional phase}

A load drop is typically observed when the debonding process has finished. At this stage the push-out force depends only on the frictional properties of the interface and the presence of interface normal pressure.

\section{Set-up of the finite element model}

ANSYS ${ }^{\circledR}$ Academic Research Mechanical, Release 17.2 was used for the finite element calculations. Figure 4 shows the finite element model of a push-out test with a specimen thickness of $300 \mu \mathrm{m}$. The model is meshed with 2D-elements with a quadratic displacement behaviour using an axisymmetric formulation (PLANE183). The bottom of the specimen holder is fixed. The displacement is applied as prescribed motion at the top of the indenter. A contact algorithm based on lagrangian multipliers is used for the contacts between indenter and fiber and between matrix and specimen holder to prevent penetration due to contact stiffness. The stiff behaviour of the specimen leads to an inaccurate displacement response during the elastic phase of the push-out test for other contact formulations because contact penetration would not be negligible compared to the indenter displacement. A criterion considering initially bonded contact with debonding is used for the connection between fiber and matrix.

\subsection{Material models}

For the rather brittle matrix material and for all other parts of the model excluding the fiber, linear-elastic material models are used. The elastic properties of all material models are summarized in Table 1 . The material model of the more ductile tungsten 


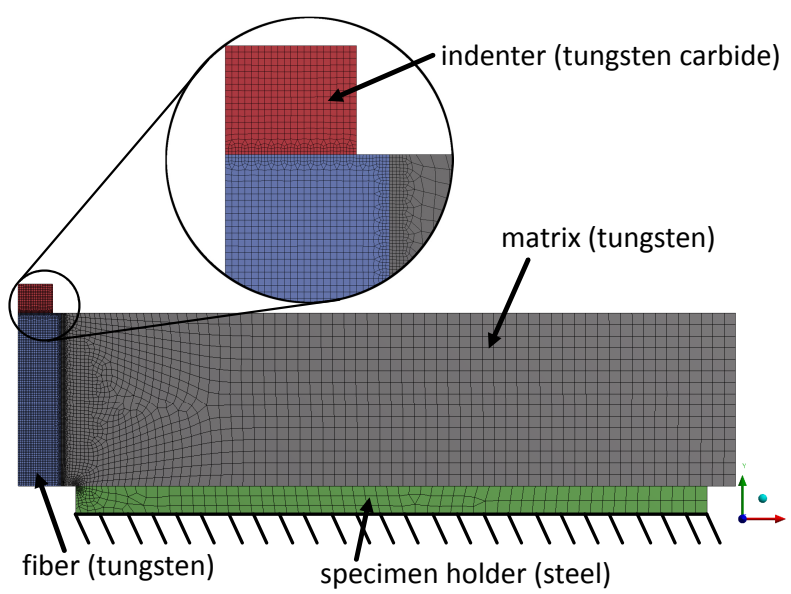

Figure 4. Finite element model of a $\mathrm{W}_{\mathrm{f}} / \mathrm{W}$ push-out test with a specimen thickness of $300 \mu \mathrm{m}$.

Table 1. Elastic properties used for the finite element calculations.

\begin{tabular}{llccl}
\hline Part & Material & Elastic modulus & Poisson's ratio & Source \\
\hline fiber, matrix & tungsten & $398 \mathrm{GPa}$ & 0.29 & {$[24]$} \\
indenter & tungsten carbide & $700 \mathrm{GPa}$ & 0.31 & {$[25]$} \\
specimen holder & steel & $200 \mathrm{GPa}$ & 0.3 &
\end{tabular}

fiber considers plasticity with isotropic hardening and a von Mises yield surface. The plastic flow curve (Figure 5) was calculated based on two stress-strain curves of tension tests given in [11].

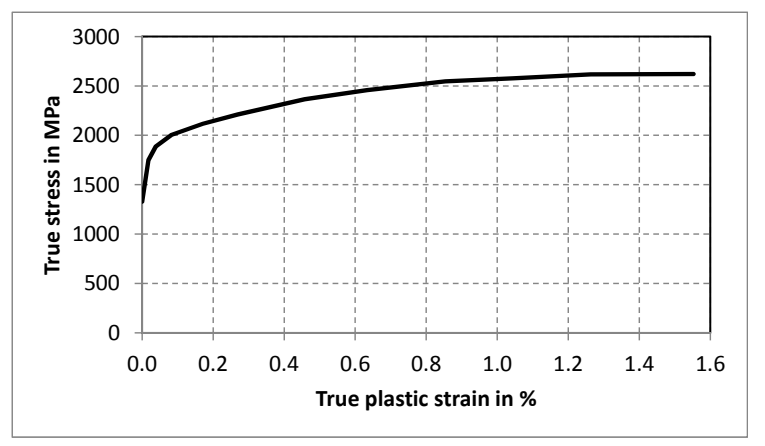

Figure 5. Flow curve determined by single wire tension test.

\subsection{Interface between fiber and matrix}

The interface layer between fiber and matrix is represented by a bonded contact. It is assumed that debonding will be dominated by shear, thus only Mode II debonding is considered and Mode I is neglected. It is modelled by a cohesive zone material model with bilinear traction separation law. Figure 6 shows the relation of tangential slip $u_{t}$ between the both sides of the interface and the shear stress $\tau$ [26].

For shear stresses smaller than the interface shear strength $\tau_{\max }$ the contact shows linear elastic behaviour with the contact stiffness $K_{t}$. When the shear stress reaches the shear strength of the interface $\left(\tau=\tau_{\max }\right)$ damage is initiated. From now on softening 


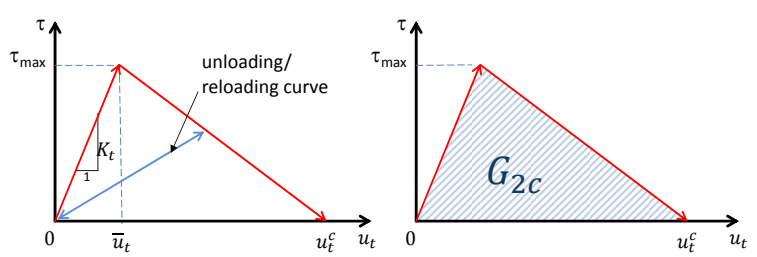

Figure 6. Traction separation behaviour of the applied cohesive zone model for the interface [26].

Table 2. Elastic properties of $\mathrm{Er}_{2} \mathrm{O}_{3}$ at room temperature [27].

\begin{tabular}{ccc}
\hline Elastic modulus & Shear modulus & Poisson's ratio \\
\hline $160 \mathrm{GPa}$ & $60 \mathrm{GPa}$ & 0.33 \\
\hline
\end{tabular}

of the contact stiffness leads to a linear decrease of the shear stress until it becomes zero at $u_{t}^{C}$ and full debonding occurs. The bonded node is released and the contact behaviour changes to frictional. As can be seen in Figure 6, the energy dissipated by the debonding process $G_{2 C}$ is

$$
G_{2 C}=\frac{1}{2} \cdot u_{t}^{C} \cdot \tau_{\max }
$$

Since no elastic properties determined for thin films of erbium oxide are available, the elastic and shear modulus for bulk material produced by sintering with a porosity of $5 \%$ is used as an approximation [27] (Table 2).

For calculating the normal stiffness $K_{n}$ of the contact an uniaxial strain state (constrained transverse strain) is assumed, which leads to the equation

$$
\sigma_{n}=\frac{(1-\nu) \cdot E}{(1+\nu) \cdot(1-2 \nu)} \cdot \varepsilon_{n}
$$

for calculating the normal stress $\sigma_{n}$ in dependence of the elastic modulus E, Poisson's ratio $\nu$ and normal strain $\varepsilon_{n}$. Together with the thickness of the interface layer $t_{i f}$ the normal stiffness can be derived from

$$
K_{n}=\frac{\sigma_{n}}{\Delta t_{i f}}=\frac{\sigma_{n}}{\varepsilon_{n} \cdot t_{i f}}=\frac{(1-\nu)}{(1+\nu) \cdot(1-2 \nu)} \cdot \frac{E}{t_{i f}} .
$$

The tangential stiffness only depends on the shear modulus $\mathrm{G}$ and the interface thickness $t_{i f}$ :

$$
K_{t}=\frac{G}{t_{i f}}
$$

\section{Choice of the interface properties for the calculations}

The aim of this work is to investigate the influence of the most important interface properties onto the push-out behaviour of $\mathrm{W}_{\mathrm{f}} / \mathrm{W}$. The first step is to find a set of interface parameters which enables the finite element model to sufficiently describe the pushout experiments of specimen manufactured by CVD with thicknesses of approximately 
Table 3. Properties of the erbium oxide interface determined by Du [14] and of the finite element model.

\begin{tabular}{|c|c|c|c|c|c|c|}
\hline \multirow{3}{*}{ Parameter } & \multirow{3}{*}{ Symbol } & \multirow{3}{*}{ Unit } & \multicolumn{4}{|c|}{ Values } \\
\hline & & & \multirow{2}{*}{ J. Du [14] } & \multicolumn{3}{|c|}{ Simulation } \\
\hline & & & & $\min$. & nominal & $\max$ \\
\hline shear strength & $\tau_{\max }$ & $\mathrm{MPa}$ & 363 & $550 / 1.5$ & 550 & $550 \cdot 1.5$ \\
\hline fracture energy (Mode II) & $G_{2 C}$ & $\mathrm{~J} / \mathrm{m}^{2}$ & 9.61 & $10 / 2$ & 10 & $10 \cdot 2$ \\
\hline coefficient of friction & $\mu$ & - & 0.64 & $0.64 / 1.5$ & 0.64 & $0.64 \cdot 1.5$ \\
\hline initial normal interface pressure & $p_{\text {ini }}$ & $\mathrm{MPa}$ & 272 & & 272 & \\
\hline
\end{tabular}

$150 \mu \mathrm{m}$ and $300 \mu \mathrm{m}$ ('nominal parameters'). In a second step this set of parameters is used as starting point for further investigations with modified parameters.

Interface properties of a $600 \mathrm{~nm}$ thick layer of erbium oxide have been determined by extensive push-out experiments and fitting of analytical models by Du [14]. Based on this work the shear strength of the interface in the finite element calculation had to be increased from $363 \mathrm{MPa}$ to $550 \mathrm{MPa}$ to achieve a similar force-displacement behaviour as observed in the experiments, as it is going to be described in the next section. The parameters from the experimental work of $\mathrm{Du}$ as well as the range of parameters that are used for the investigations with the finite element method are summarized in Table 3 .

\section{Simulation of push-out test with nominal parameters}

Figure 7 shows that the force-displacement behaviour of the finite element model with nominal interface parameters represents well the experiments with specimen thicknesses of approximately $150 \mu \mathrm{m}$ and $300 \mu \mathrm{m}$. Due to sudden elastic unloading of the test machine after debonding no force data was recorded for the dotted portions of the experimental curves. Details on the sample production and experiments can be found in $[14]$.

As can be seen in Figure 7a, the experimental curves of two out of three samples of $150 \mu \mathrm{m}$ thick CVD-specimen show a sudden force-drop at debonding and do not show any evidence for an initiation of debonding before reaching the maximum force. One sample (CVD Du, $\mathrm{t}=0.147$ ) shows softening before completion of debonding. The maximum forces are between $25 \mathrm{~N}$ and $28 \mathrm{~N}$. The maximum force of the finite element calculation is near the upper bound of the experiments whereas the force during the frictional phase of the test is at the lower bound.

The two specimen with a thickness of $300 \mu \mathrm{m}$ show significant differences regarding force values and qualitative shape of the force-displacement curve (Figure 7b). One sample ('CVD Du, $\mathrm{t}=0.293$ ') behaves qualitatively comparable with the thinner $150 \mu \mathrm{m}$ samples. The other one ('CVD Du, $\mathrm{t}=0.309$ ') reaches higher forces and behaves differently. Its force displacement curve shows a distinctive softening for forces higher than the maximum force of the sample mentioned before.

While experiments allow only an external observation during the test, finite element calculations can give a more detailed view inside the interface, e.g. the stress state and the debonding status. Figure 8 shows again the calculated force-displacement curves for both specimen thicknesses. The elastic stiffness slope in the diagram and the plot of the derivative of the force with respect to the indenter displacement $\mathrm{d} F / \mathrm{d} s$ help to visualize deviations from linear elastic behaviour. The thinner specimen shows ideally elastic behaviour until a very short debonding phase which can only be identified in 


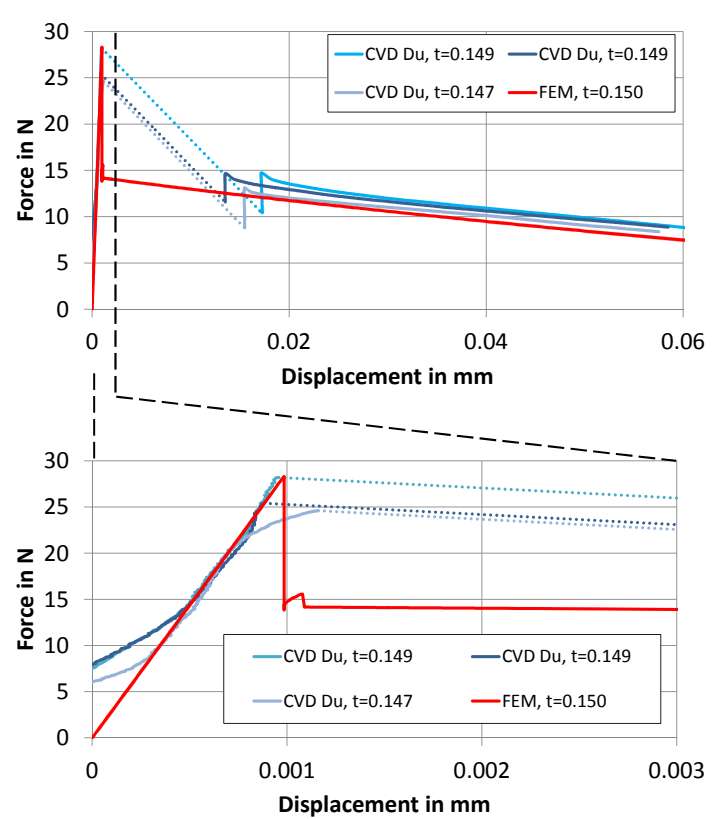

(a) specimen thickness $\approx 150 \mu \mathrm{m}$

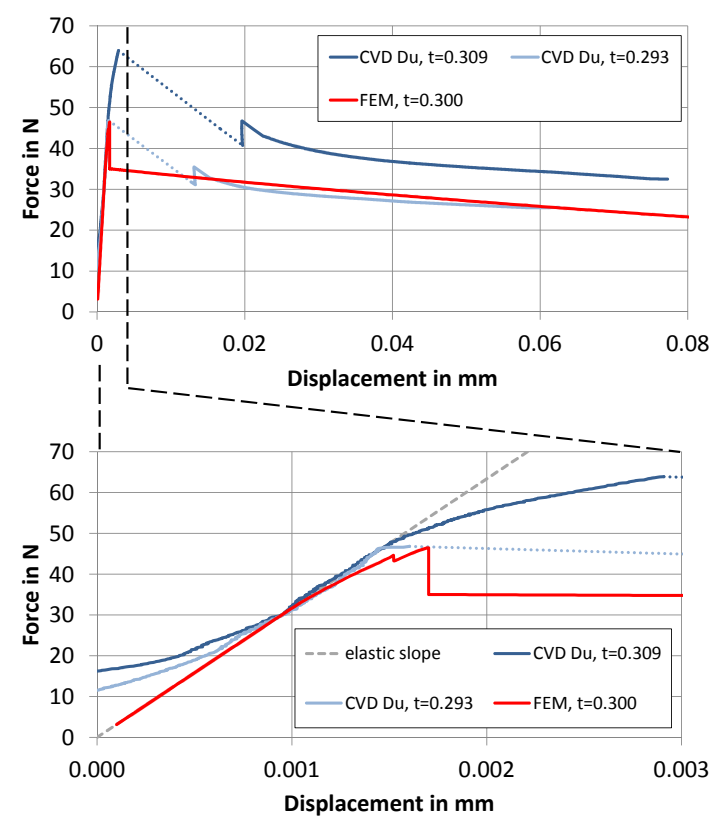

(b) specimen thickness $\approx 300 \mu \mathrm{m}$

Figure 7. Experimental push-out curves of specimen produced by CVD compared with finite-element calculations with nominal parameters shown in Table 3 (lower graphs: detail views of upper graphs). Due to sudden elastic unloading of the test machine after debonding no force data was recorded for the dotted portions of the experimental curves.

the derivative (Figure 8a, lower graph) and in the plot of the percentage of debonded interface (Figure 8a, upper graph). In contrast to this the thicker specimen shows a more gradual debonding. In Figure $8 \mathrm{~b}$ it can be seen clearly that the stiffness of the specimen decreases significantly as soon as debonding is initiated at $1 \mu \mathrm{m}$ of indenter displacement. In addition, a slight decrease of the specimen stiffness can be observed in the derivative at $0.8 \mu \mathrm{m}$, which is caused by minor plastic indentation into the fiber and which does not have a significant contribution to the total displacement.

The stress state of the interface is shown for three stages (not debonded, partially debonded, fully debonded), which are marked in the force-displacement curves in Figure 8 and for both specimen thicknesses in Figures 9 and 10. For stage II (partially debonded interface) the debonded regions of the interface can be clearly identified by the distribution of the shear stress. Despite the initial interface pressure of $272 \mathrm{MPa}$ the interface shows tensile normal stresses at the bottom of the thinner specimen due to bending. The stress distribution of stages I and III are quite similar, although the debonded interface at stage III is not able to transfer tensile normal stresses. All stages have in common that the stress peaks at the top of the specimens are more distinct for the thicker samples.

\section{Influence of the interface parameters on the push-out behaviour}

Scaling the interface parameters shear strength $\left(\tau_{\max }\right)$, coefficient of friction $(\mu)$, fracture energy of Mode II $\left(G_{2 C}\right)$ as well as the true stress of the plastic flow curve $\left(\sigma_{\text {true }}\right.$, see Figure 5) will give a better understanding of how the force-displacement curve, which is the basis for interpreting the experiments, is influenced by them. 

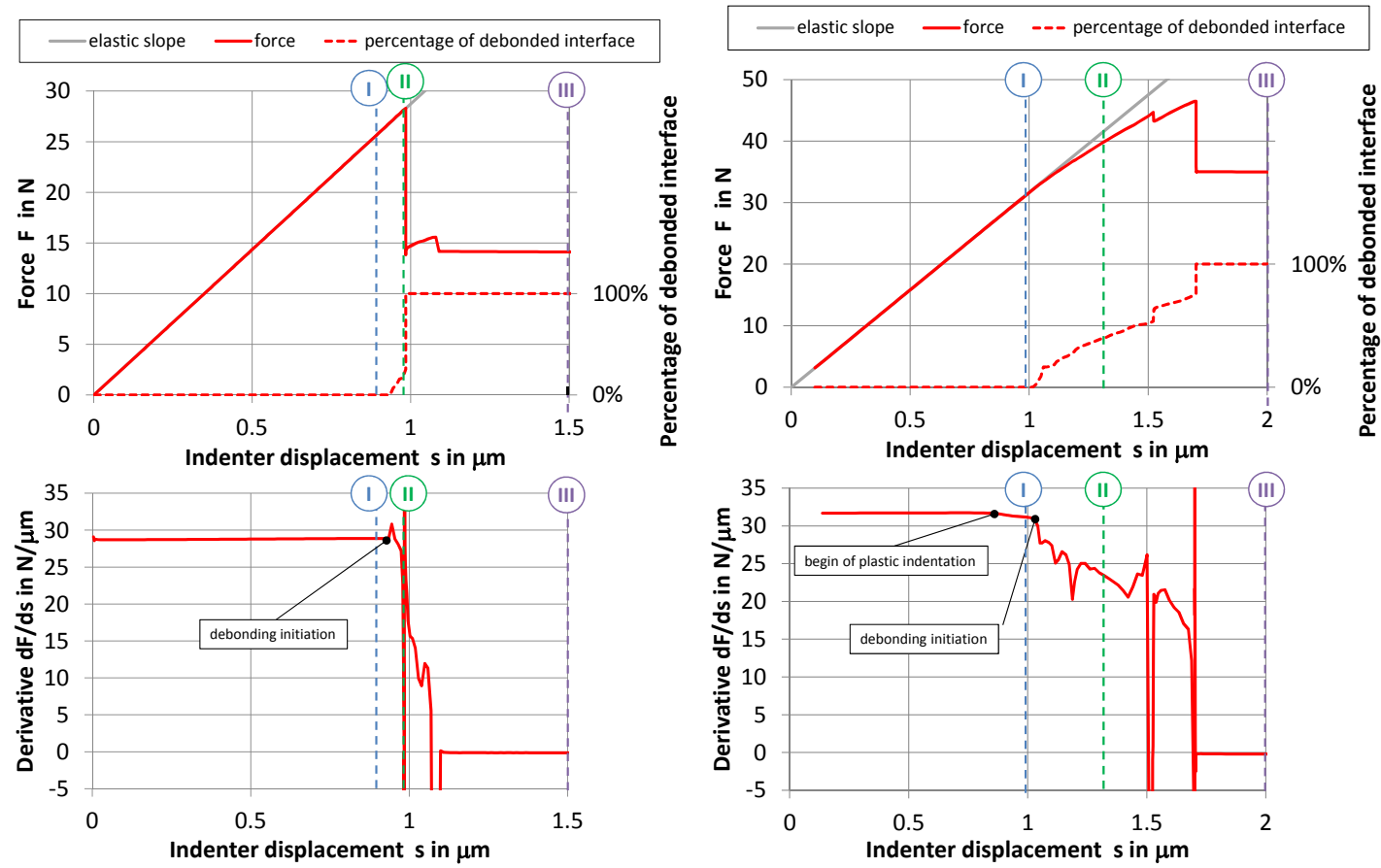

(a) specimen thickness $150 \mu \mathrm{m}$

(b) specimen thickness $300 \mu \mathrm{m}$

Figure 8. Calculated indenter force, percentage of debonded interface and tangential stiffness of specimen plotted vs. indenter displacement.
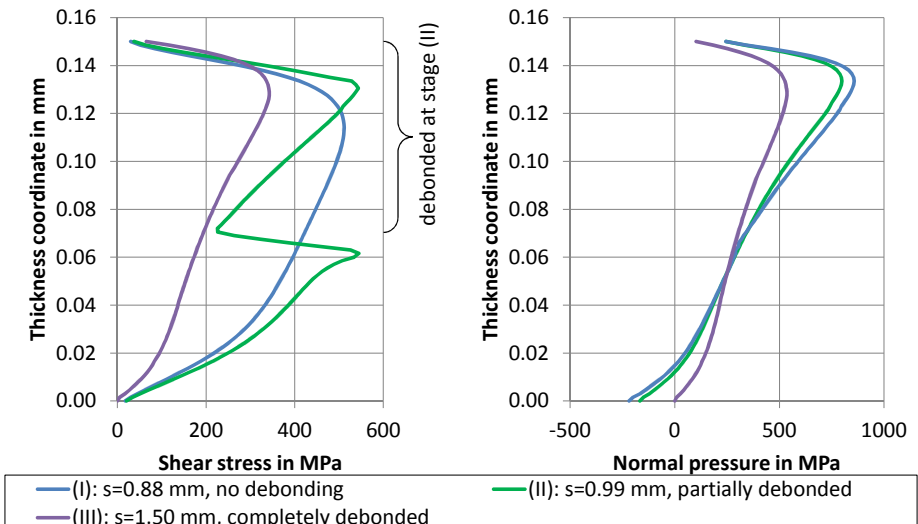

specimen thickness $150 \mu \mathrm{m}$

Figure 9. Calculated stress state of the interface (nominal interface parameters, specimen thickness $150 \mu \mathrm{m}$ ). The corresponding stages I-III are marked in the force-displacement curve in Figure 8a. 

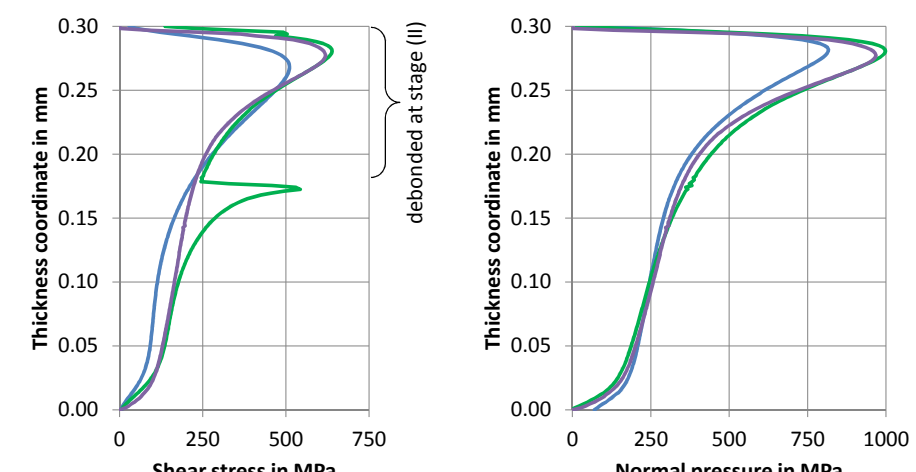

-(I): $\mathrm{s}=0.96 \mathrm{~mm}$, no debonding
-(III): $\mathrm{s}=1.99 \mathrm{~mm}$, completely debonded

Normal pressure in $\mathrm{MPa}$

specimen thickness $300 \mu \mathrm{m}$

Figure 10. Calculated stress state of the interface (nominal interface parameters, specimen thickness $300 \mu \mathrm{m}$ ). The corresponding stages I-III are marked in the force-displacement curve in Figure 8b.

\subsection{Shear strength $\tau_{\max }$}

The nominal value of the shear strength $\left(\tau_{\max }=550 \mathrm{MPa}\right)$ is divided $/$ multiplied by the factor 1.5 to investigate it's influence. Figure 11 shows the calculated forcedisplacement curves for the different values of $\tau_{\max }$. For the thinner specimen the force at debonding initiation and the achieved maximum force show a behaviour proportional to the shear strength. For the thicker specimen this is only the case for the debonding initiation. The dependency of the maximum force is less than proportional.
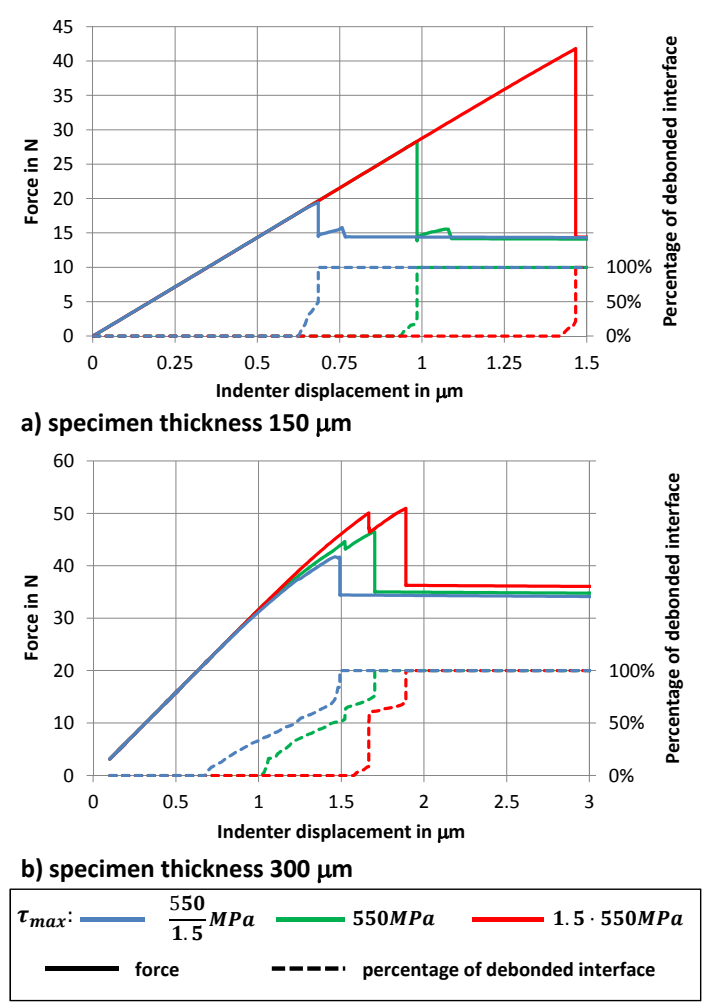

Figure 11. Influence of the shear strength $\tau_{\max }$ on the push-out behaviour for specimen thicknesses of a) $150 \mu \mathrm{m}$ and b) $300 \mu \mathrm{m}$. 


\subsection{Coefficient of friction $\mu$}

As expected, the coefficient of friction $\mu$ influences directly the force level during the frictional phase after completion of the debonding. Furthermore it plays an important role during the debonding process itself. Figure 12 shows the calculated force-displacement curves for different coefficients of friction. A small coefficient $(0.64 / 1.5 \approx 0.43)$ leads to a sudden debonding after its initiation while increased friction extends the length of the debonding phase from $0.7 \mu \mathrm{m}$ to $1.9 \mu \mathrm{m}$ of indenter displacement for the specimen with $300 \mu \mathrm{m}$ thickness. The same effect can be noted for the thinner specimen, where it is less distinct. During the debonding process frictional sliding of the debonded portion of the interface is necessary to be able to redistribute the load to areas that are still bonded. Higher coefficients of friction increase the shear loads that can be carried by the debonded areas due to the initial and the additional normal pressure caused by the transverse strain of the fiber.
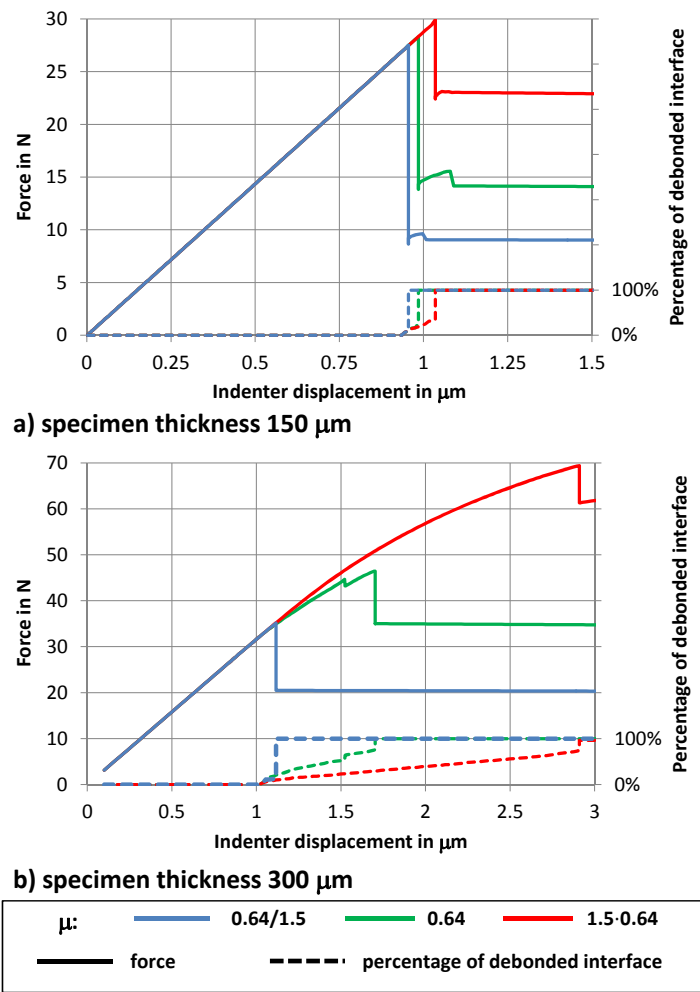

Figure 12. Influence of the coefficient of friction $\mu$ on the push-out behaviour for specimen thicknesses of a) $150 \mu \mathrm{m}$ and b) $300 \mu \mathrm{m}$.

\subsection{Fracture energy $G_{2 C}$}

The influence of the fracture energy of the interface for Mode II-debonding depends strongly on the thickness of the specimen (Figure 13). Even though its value of $10 \mathrm{~J} / \mathrm{m}^{2}$ was multiplied and divided, respectively, with the factor 2 , there was no significant influence on the resulting force-displacement curve of the thinner specimen with a thickness of $150 \mu \mathrm{m}$. For the thicker specimen increasing the fracture energy leads to an extension whereas decreasing leads to a reduction of the debonding phase with regard to indenter displacement. 

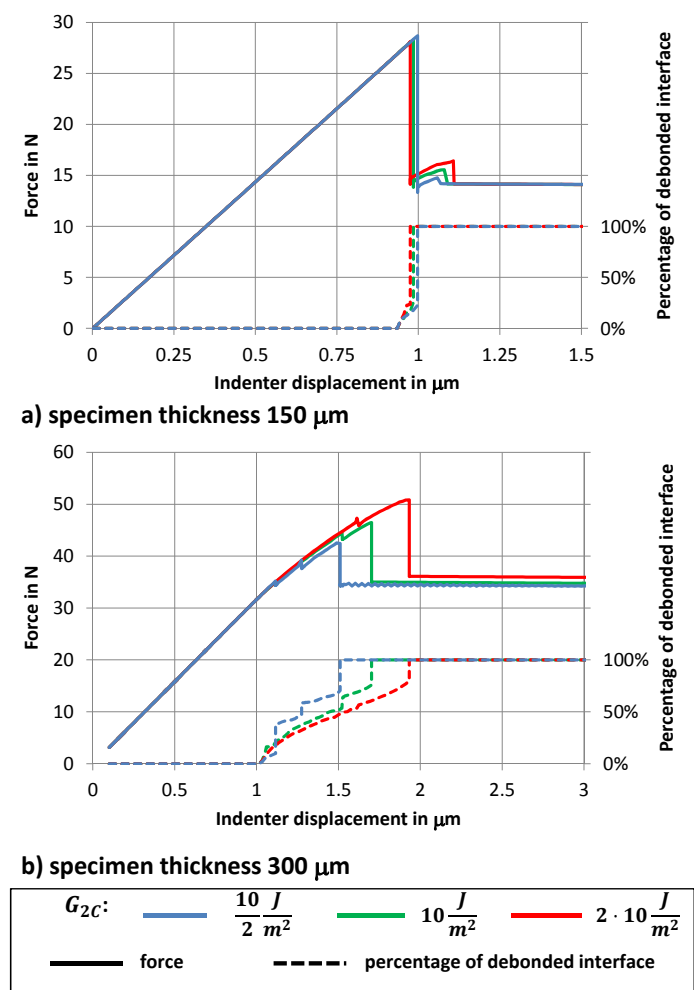

Figure 13. Influence of the fracture energy (Mode II) $G_{2 C}$ on the push-out behaviour for specimen thicknesses of a) $150 \mu \mathrm{m}$ and b) $300 \mu \mathrm{m}$.

\subsection{Flow curve of the fiber}

Besides the flow curve shown in Figure 5 the push-out tests were calculated with different plastic behaviours of the fiber. On the one hand, linear elastic material behaviour without plasticity was assumed as material model and on the other hand the true stress of the flow curve $\sigma_{\text {true }}$ was reduced by the "flow stress reduction factor" 1.2 and 2.0, respectively (Figure 14). The plasticity of the fiber can lead to its plastic indentation. Table 4 gives an overview of the depth of the plastic indentations for both specimen thicknesses and all flow curve reduction factors. Due to the lower forces the thinner specimen shows only significant indentation for the calculation with the flow stress reduction factor 2. For this case the indenter displacement at debonding initiation is shifted by around $0.1 \mu \mathrm{m}$, the debonding progress is slightly prolongated and the frictional force is marginally increased as shown by the orange lines in Figure 14(a). The sensitivity of the thicker specimen regarding the flow curve is much higher. Even with the nominal flow curve, which shows a small indentation of $0.11 \mu \mathrm{m}$, the frictional forces are higher compared to the linear elastic fiber. Force decrease of the flow stress leads to an amplification of this effect. For the flow stress reduction factor of $x=2$ the indentation becomes excessive $(36 \mu \mathrm{m})$ and the debonding process needs $34 \mu \mathrm{m}$ of indenter displacement from the initiation to completion. Also the obtained maximum force is lower (orange lines in Figure 14(b)). It is worth to mention that the debonding of the interface and the forming of the indentation is not finished after the force drop following the maximum force at an indenter displacement of $2.6 \mu \mathrm{m}$. Figure 15 shows the deformed finite element model at the end of this calculation. 


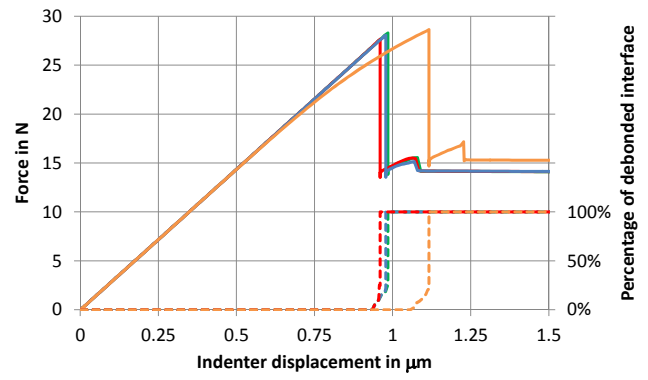

a) specimen thickness $150 \mu \mathrm{m}$

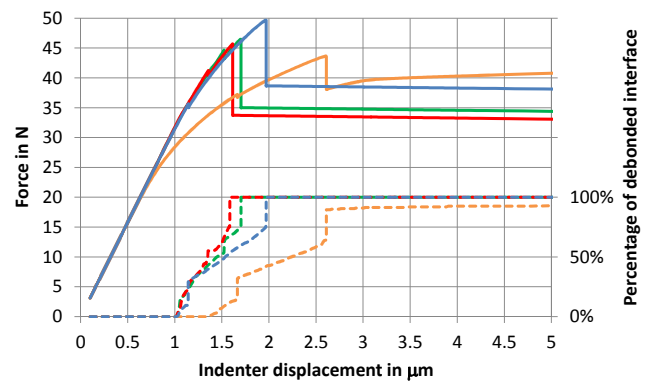

b) specimen thickness $300 \mu \mathrm{m}$

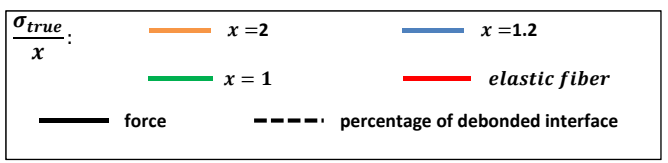

Figure 14. Influence of scaling the true stress of the flow curve of the fiber by the flow stress reduction factor $\mathrm{x}$ on the push-out behaviour for specimen thicknesses of a) $150 \mu \mathrm{m}$ and b) $300 \mu \mathrm{m}$.

Table 4. Depth of plastic indentation with different flow curves of the tungsten fiber. The true stress of the nominal flow curve $\sigma_{\text {true }}$ is reduced by the reduction factor $\mathrm{x}$.

\begin{tabular}{clc}
\hline Flow stress & \multicolumn{2}{c}{ Specimen thickness } \\
\cline { 2 - 3 } reduction factor $\mathbf{x}$ & $\mathbf{1 5 0} \boldsymbol{\mu m}$ & $\mathbf{3 0 0} \boldsymbol{\mu m}$ \\
\hline 1 (nominal) & $0.00 \mu \mathrm{m}$ & $0.11 \mu \mathrm{m}$ \\
1.2 & $0.02 \mu \mathrm{m}$ & $0.37 \mu \mathrm{m}$ \\
2 & $0.24 \mu \mathrm{m}$ & $36.0 \mu \mathrm{m}$ \\
\hline
\end{tabular}

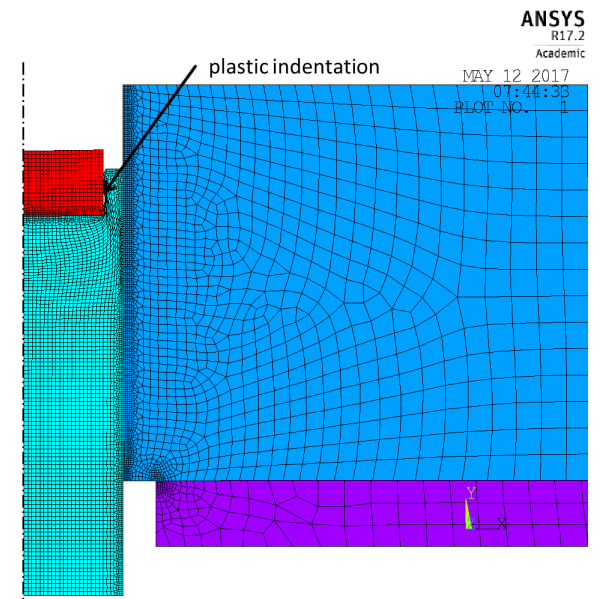

Figure 15. Deformation of the calculated push-out test with large plastic indentation (specimen thickness: $300 \mu \mathrm{m}$, flow stress reduction factor $\mathrm{x}=1 / 2$, see section 8.4). 


\section{Discussion}

Based on experimentally derived interface properties a finite element model of a pushout test of a single fiber composite has been set up. It is able to appropriately capture the experimental force-displacement behaviour during the push-out test of tungsten fiber-reinforced tungsten $\left(\mathrm{W}_{\mathrm{f}} / \mathrm{W}\right)$ with an interface of erbium oxide with a thickness of $600 \mathrm{~nm}$, a fiber diameter of $150 \mu \mathrm{m}$ and a tungsten matrix produced by chemicalvapour-deposition (CVD). This confirms that these parameters, which originate from the work of $\mathrm{Du}[14]$, are within a reasonable range and that the finite element model is able to capture the most important physical effects that are acting during the push-out test. The interface shear strength $\tau_{\max }$ was the only parameter that was significantly modified for the calculation model. Its value was increased from $363 \mathrm{MPa}$ to $550 \mathrm{MPa}$ to achieve a proper value for the maximum forces. The shear strength can be interpreted as an effective shear strength which leads to a consistent behaviour between simulation model and experiments for the investigated set up. While the existence and source of initial interface pressure in $\mathrm{W}_{\mathrm{f}} / \mathrm{W}$ produced by CVD is not clarified yet, consideration of the analytically derived values of $\mathrm{Du}$ within the finite element calculations leads to realistic results.

The calculations show that friction not only plays an important role during the purely frictional part of the experiment after completion of debonding. It also influences strongly the progress of debonding due to the presence of normal interface pressure and its amplification due to the elastic transverse strain of the fiber. This is particularly the case for thicker specimens, which contain more interface area and therefore can transmit higher loads by friction.

As soon as small plastic indentations occur, the results are influenced by a changed frictional behaviour. This is particularly the case for thicker specimens which need higher forces for the push-out. The additional plastic transverse strain is irreversible and even remains after reduction of the indenter load after debonding. The investigation of the same set of parameters with differently scaled flow curves compared to a fiber with linear-elastic behaviour showed that the debonding progress is also influenced. A plastic softening of the fiber material comes along with an extension of the debonding phase. In the physical experiment the initiation of debonding cannot be detected on the basis of the beginning of the nonlinear force-displacement behaviour if plastic indentations are present. Plastic indentations cause higher forces in the frictional phase of the experiment, which can lead to misinterpretations if this is not taken into account properly. Therefore it must be recommended not to calculate interface parameters with the help of analytical models based on push-out tests that exhibit plastic indentations. This makes it also difficult to investigate $\mathrm{W}_{\mathrm{f}} / \mathrm{W}$ with push-out tests if the fiber is softened due to the preparation history or heat treatment. This phenomenon can be observed for thicker samples produced by HIP (hot isostatic pressing). Figure 16 shows a cross section polish of a pushed HIP-sample with a thickness of approximately $300 \mu \mathrm{m}$. With a maximum force of $55 \mathrm{~N}$ the indenter created a plastic indentation with a depth of approximately $70 \mu \mathrm{m}$. As can be seen in Figure 17 the shape of the nonlinear portion of the force displacement curve before reaching the maximum force corresponds well to the calculation with a flow stress reduction factor of 2 (see Section 8.4), whereas the maximum force and the indentation depth of the calculation are lower.

Thin specimens show a very abrupt debonding behaviour which simplifies the identification of debonding initiation - it approximately appears at the maximum force. Furthermore the influence of the fracture energy of Mode II on the force-displacement 


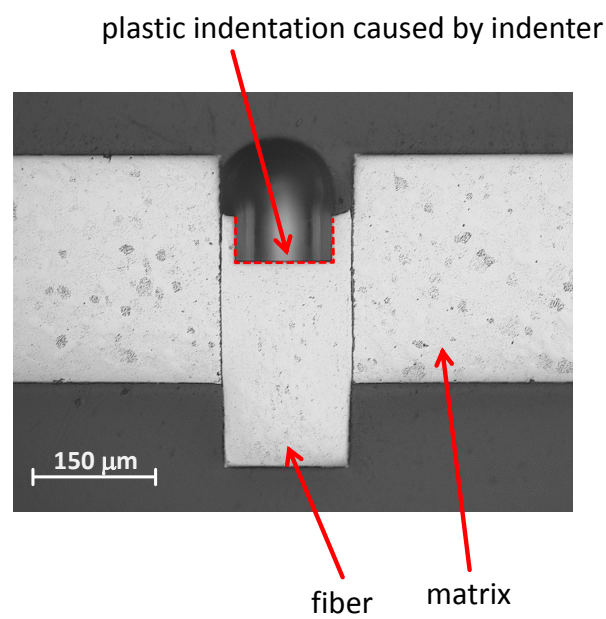

Figure 16. Cross-section polish of HIP specimen with a thickness of $\approx 300 \mu \mathrm{m}$ after the push-out test.

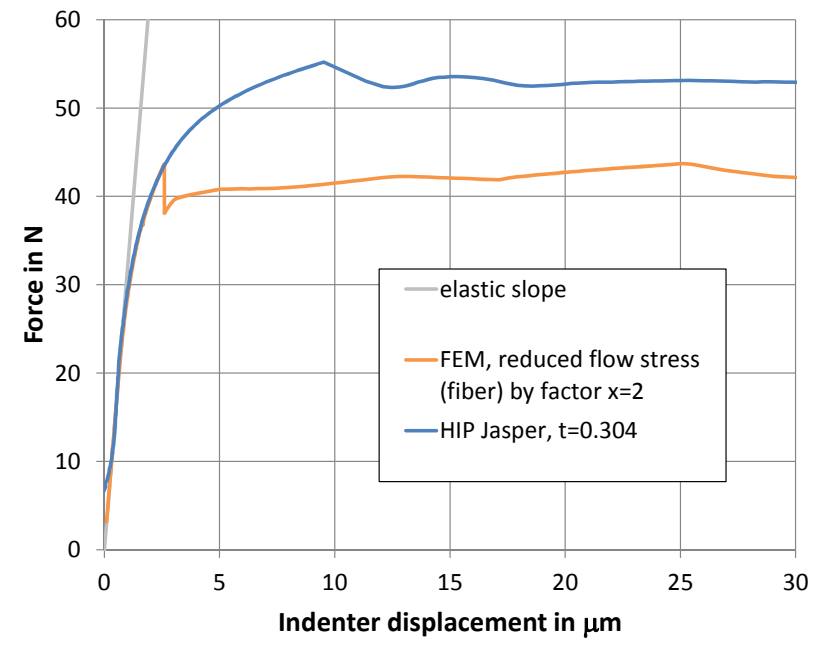

Figure 17. Force-displacement curve of HIP-sample shown in Figure 16 compared to the finite element calculation with flow curve reduction factor of 2 as described in Section 8.4. 
curve is negligible. Due to the lower forces needed for the initiation of debonding plastic indentations are not an issue. Nevertheless care has to be taken when interpreting the results of thin specimen. The bending of the specimen lying on the holder can lead to normal tensile stresses which can initiate debonding under Mode I at the bottom of the specimen. Debonding under Mode I was not considered in the calculation model because properties of the interface for Mode I (strength, fracture energy) were not available. It is hard to estimate how debonding initiation at the bottom of a specimen would influence the results. Therefore it is recommended to keep the hole diameter of the specimen holder as small as possible.

\section{Outlook}

The frictional stage of the force displacement curve of $\mathrm{W}_{\mathrm{f}} / \mathrm{W}$ strongly suggests the existence of initial normal interface pressure between fiber and matrix. Nevertheless the existence of residual stresses of $\mathrm{W}_{\mathrm{f}} / \mathrm{W}$ produced by CVD could neither be explained nor be verified satisfactorily. To clarify the presence of residual stresses in $\mathrm{W}_{\mathrm{f}} / \mathrm{W}$ originating from different production routes measurements with synchrotron tomography have been performed at the European Synchrotron Radiation Facility (ESRF) in Grenoble.

Push-out tests with $\mathrm{W}_{\mathrm{f}} / \mathrm{W}$ are underlying limitations regarding the thickness of the specimen. The results of thin specimen might be influenced by strong bending and thus tensile interface stresses in normal direction at the bottom of the specimen. On the other hand thick specimens need higher forces for the push-out which can lead to plastic indentations that make the interpretation of the experiment hardly impossible. In contrast to the push-out test the pull-out test is closer to the situation that is present if a crack is bridged by fibers. Therefore activities to investigate $\mathrm{W}_{\mathrm{f}} / \mathrm{W}$ by means of pull-out tests have been initiated.

Multi-fiber samples produced by chemical vapour infiltration (CVI) showed extrinsic toughening during three-point-bending tests [8]. While simulation of multi-fiber systems would be of great interest to create a deeper understanding of the acting mechanisms several hurdles have to be taken to put it into practice in future work: Changing the element formulation from axisymmetric 2D to 3D leads to a significant increase of active degrees of freedom in the model. Together with the huge number of iterations of the finite-element solver that are necessary to simulate the debonding process this will lead to very long calculation times. Furthermore an approach for modelling the crack propagation in the matrix material needs to be validated.

\section{Acknowledgement}

This work has been carried out within the framework of the EUROfusion Consortium and has received funding from the Euratom research and training programme 20142018 under grant agreement No 633053. The views and opinions expressed herein do not necessarily reflect those of the European Commission. 


\section{References}

[1] Bachmann C, Aiello G, Albanese R, et al. Initial DEMO tokamak design configuration studies. Fusion Eng Des. 2015;98-99:1423 - 1426. Proceedings of the 28th Symposium On Fusion Technology (SOFT-28).

[2] Philipps V. Tungsten as material for plasma-facing components in fusion devices. J Nucl Mater. 2011;415(1, Supplement):S2 - S9. Proceedings of the 19th International Conference on Plasma-Surface Interactions in Controlled Fusion.

[3] Coenen J, Antusch S, Aumann M, et al. Materials for DEMO and reactor applicationsboundary conditions and new concepts. Phys Scr. 2015;2016(T167):014002.

[4] Roth J, Tsitrone E, Loarte A, et al. Recent analysis of key plasma wall interactions issues for ITER. J Nucl Mater. 2009;390:1 - 9. Proceedings of the 18th International Conference on Plasma-Surface Interactions in Controlled Fusion Device.

[5] Zinkle S, Ghoniem N. Operating temperature windows for fusion reactor structural materials. Fusion Eng Des. 2000;51-52:55 - 71.

[6] Bolt H, Barabash V, Federici G, et al. Plasma facing and high heat flux materials needs for ITER and beyond. J Nucl Mater. 2002;307-311, Part 1:43 - 52.

[7] Launey ME, Ritchie RO. On the fracture toughness of advanced materials. Adv Mater. 2009;21(20):2103-2110.

[8] Riesch J, Höschen T, Linsmeier C, et al. Enhanced toughness and stable crack propagation in a novel tungsten fibre-reinforced tungsten composite produced by chemical vapour infiltration. Phys Scr. 2014;2014(T159):014031.

[9] Wachtman JB, Cannon WR, Matthewson MJ. Mechanical properties of ceramics. John Wiley \& Sons; 2009.

[10] Evans AG. Perspective on the development of high-toughness ceramics. J Am Ceram Soc. 1990;73(2):187-206.

[11] Riesch J, Buffiere JY, Höschen T, et al. In situ synchrotron tomography estimation of toughening effect by semi-ductile fibre reinforcement in a tungsten-fibre-reinforced tungsten composite system. Acta Mater. 2013;61(19):7060-7071.

[12] Riesch J, Han Y, Almanstötter J, et al. Development of tungsten fibre-reinforced tungsten composites towards their use in DEMO - Potassium doped tungsten wire. Phys Scr. 2016; (T167):014006.

[13] Bogetti TA, John W Gillespie J. Process-induced stress and deformation in thick-section thermoset composite laminates. J Compos Mater. 1992;26(5):626-660.

[14] Du J. A feasibility study of tungsten-fiber-reinforced tungsten composites with engineered interfaces [dissertation]. Universität München; 2011.

[15] Jasper B, Schönen S, Du J, et al. Behavior of tungsten fiber-reinforced tungsten based on single fiber push-out study. Nucl Mater Energy. 2016;9:416 - 421.

[16] Liang C, Hutchinson J. Mechanics of the fiber pushout test. Mech Mater. 1993;14(3):207221.

[17] Shetty DK. Shear-lag analysis of fiber push-out (indentation) tests for estimating interfacial friction stress in ceramic-matrix composites. J Am Ceram Soc. 1988;71(2):C-107C-109.

[18] Greszczuk L. Theoretical studies of the mechanics of the fiber-matrix interface in composites. In: Interfaces in composites. ASTM International; 1969.

[19] Lawrence P. Some theoretical considerations of fibre pull-out from an elastic matrix. J Mater Sci. 1972;7(1):1-6.

[20] Du J, Höschen T, Rasinski M, et al. Feasibility study of a tungsten wire-reinforced tungsten matrix composite with zrox interfacial coatings. Compos Sci Technol. 2010;70(10):1482 -1489 .

[21] Du J, Höschen T, Rasinski M, et al. Shear debonding behavior of a carbon-coated interface in a tungsten fiber-reinforced tungsten matrix composite. J Nucl Mater. 2011;417(1):472 - 476. Proceedings of ICFRM-14.

[22] Mao Y, Coenen J, Riesch J, et al. Influence of the interface strength on the mechanical 
properties of discontinuous tungsten fiber-reinforced tungsten composites produced by field assisted sintering technology. Composites, Part A. 2018;107:342 - 353.

[23] Chandra N, Ghonem H. Interfacial mechanics of push-out tests: theory and experiments. Composites, Part A. 2001;32(3-4):575 - 584.

[24] ITER material properties handbook (IMPH). Appendix A, Materials Design Limit Data. ITER Document No. 222RLN and G 74 MA 8 01-05-28 W 0.2. ; 2013.

[25] Kurlov AS, Gusev AI. Tungsten carbides. Vol. 184. Springer; 2013.

[26] ANSYS, Release 17.2, Help System, Mechanical APDL - Theory Reference, ANSYS Inc.; 2017.

[27] Manning WR, Hunter O. Elastic properties of polycrystalline yttrium oxide, holmium oxide, and erbium oxide: High-temperature measurements. J Am Ceram Soc. 1969; 52(9):492-496. 